\title{
High-efficiency liposomal encapsulation of a tyrosine kinase inhibitor leads to improved in vivo toxicity and tumor response profile
}

This article was published in the following Dove Press journal:

International Journal of Nanomedicine

18 October 2013

Number of times this article has been viewed

\author{
Rajesh Mukthavaram' \\ Pengfei Jiang' \\ Rohit Saklecha' \\ Dmitri Simberg ${ }^{3,4}$ \\ Ila Sri Bharati' \\ Natsuko Nomura' \\ Ying Chao' \\ Sandra Pastorino' \\ Sandeep C Pingle' \\ Valentina Fogal' \\ Wolf Wrasidlo',2 \\ Milan Makale ${ }^{1,2}$ \\ Santosh Kesari ${ }^{1,2}$ \\ 'Translational Neuro-Oncology \\ Laboratories, ${ }^{2}$ Department of \\ Neurosciences, ${ }^{3}$ Solid Tumor \\ Therapeutics Program, Moores \\ Cancer Center, UC San Diego, \\ La Jolla, CA, ${ }^{4}$ Skaggs School of \\ Pharmacy and Pharmaceutical \\ Sciences, University of Colorado, \\ Denver, CO, USA
}

Correspondence: Santosh Kesari Moores Cancer Center, UC San Diego, 3855 Health Sciences Drive, MC\#0819, La Jolla, CA 92093-0819, USA

Tel + I 8588227524

$\mathrm{Fax}+\mathrm{I} 8588223033$

Email skesari@ucsd.edu

Milan Makale

Moores Cancer Center, UC San Diego, 3855 Health Sciences Drive, MC\#0819,

La Jolla, CA 92093-0819, USA

Tel +l 8588226922

Fax + I 8588220022

Emailmmakale@ucsd.edu

\begin{abstract}
Staurosporine (STS) is a potent pan-kinase inhibitor with marked activity against several chemotherapy-resistant tumor types in vitro. The translational progress of this compound has been hindered by poor pharmacokinetics and toxicity. We sought to determine whether liposomal encapsulation of STS would enhance antitumor efficacy and reduce toxicity, thereby supporting the feasibility of further preclinical development. We developed a novel reverse pH gradient liposomal loading method for STS, with an optimal buffer type and drug-to-lipid ratio. Our approach produced $70 \%$ loading efficiency with good retention, and we provide, for the first time, an assessment of the in vivo antitumor activity of STS. A low intravenous dose $(0.8 \mathrm{mg} / \mathrm{kg})$ inhibited U87 tumors in a murine flank model. Biodistribution showed preferential tumor accumulation, and body weight data, a sensitive index of STS toxicity, was unaffected by liposomal STS, but did decline with the free compound. In vitro experiments revealed that liposomal STS blocked Akt phosphorylation, induced poly(ADP-ribose) polymerase cleavage, and produced cell death via apoptosis. This study provides a basis to explore further the feasibility of liposomally encapsulated STS, and potentially related compounds for the management of resistant solid tumors.
\end{abstract}

Keywords: liposomes, staurosporine, glioblastoma, biodistribution, efficacy

\section{Introduction}

Staurosporine (STS) is a pan-kinase inhibitor that exhibits potent activity against an array of cancer types in vitro. ${ }^{1-5}$ Even multidrug resistant tumor cells are rapidly driven into apoptosis, which has prompted the evaluation of STS for clinical use. ${ }^{6,7}$ However, translational development has not proved feasible because of poor pharmacokinetics and toxicity. ${ }^{6,8}$ A number of less potent but more stable STS analogs are in clinical trials, but these too are constrained by toxicity, which includes hyperglycemia with acidosis, pulmonary dysfunction, hypotension, creatinine elevation, diarrhea, nausea, and vomiting. ${ }^{9,10}$ Our aim was to assess whether liposomal encapsulation of STS would reduce toxicity and allow a comparatively low systemic dose to exert a significant antitumor effect in vivo.

The mechanism by which STS induces apoptosis is incompletely understood, but it is known that this compound inhibits multiple signaling pathways central to normal and tumor cell survival. STS, to a large extent, exerts its effects by interfering with phosphorylation of protein kinase $\mathrm{B}(\mathrm{Akt})$, which is a key convergence node for cytokine signaling and apoptosis pathways. ${ }^{11-13}$ Other members of the protein kinase family of signaling proteins, ie, protein kinase $\mathrm{C}$, protein kinase $\mathrm{A}$, and protein kinase $\mathrm{G}$, also have important roles in tumorigenesis in different cancer types, including 
glioblastoma, and are inhibited by STS. ${ }^{14-17}$ Further, STS induces caspase-3-dependent apoptotic cell death through cleavage of several key proteins, such as poly(ADP-ribose) polymerase (PARP) $1 .{ }^{2,18-20}$ These interactions may account for the ability of STS to induce apoptosis in chemotherapyresistant cancer cells, a characteristic that continues to elicit interest from a therapeutic perspective.

Liposomal encapsulation of STS has been proposed because this mode of drug delivery can reduce toxicity by substantially reducing normal tissue exposure..$^{21,22}$ The liposomal bilayer would be expected to shield STS from plasma proteins, which tend to bind STS and reduce its circulation half-life. Optimal liposomal sizing (50-200 nm) combined with polyethylene glycol (PEG) conjugation to the external liposomal surface markedly slows circulatory clearance. ${ }^{23,24}$ Moreover, leaky microvasculature at tumor and metastatic sites facilitates preferential accumulation of liposomes, resulting in localization of drug to attain therapeutic concentrations in tumor tissue, even with a low intravenous systemic dose. ${ }^{25-27}$

While an attractive option, liposomal encapsulation of the 7-hydroxy STS analog (UCN-01), has so far been quite limited in terms of loading efficiency. ${ }^{28,29}$ Hence, before conducting in vivo experiments with liposomal STS, we required a strategy by which this compound could be efficiently and stably encapsulated. We developed a novel remote loading approach modified from existing $\mathrm{pH}$ gradient methodology and utilizing optimized buffer composition. The final STS loading produced a drug-to-lipid ratio of 0.09 (mole/mole), compared with 0.03 for the 7-hydroxy STS analog, which is the highest previously reported in the literature for an STS class compound. ${ }^{28,29}$

Our goal here was to demonstrate the feasibility of liposomal STS as a potential cancer therapeutic. We utilized a resistant, fast-growing human U87 tumor murine flank model to test the hypothesis that liposomal encapsulation of STS reduces its systemic toxicity and facilitates effective antitumor activity in vivo. We also sought to investigate and compare the tumor cell killing mechanism of free and liposomal STS. Our study may provide a basis for further preclinical research exploring liposomally delivered STS and its analogs for managing multiple types of resistant solid cancers.

\section{Materials and methods Animal subjects}

Athymic nu/nu mice of either gender and 5-6 weeks of age were used, with five mice housed in sterilized cages supplied with purified air passed through activated charcoal and HEPA filters. The mice were provided with autoclaved bedding, food, and water. All animal procedures were conducted in strict accordance with all appropriate regulatory standards under UC San Diego animal use protocol \#S10057, which was reviewed and approved by the UC San Diego Institutional Animal Care and Use Committee.

\section{Cell culture and reagents}

A172, U87, U118, and U251 glioblastoma lines, a PC3 pancreatic cancer line, a B16F10 melanoma line, and a MDAMB-231 breast cancer line were maintained under standard culture conditions in Dulbecco's Modified Eagle's Medium supplemented with $10 \%$ fetal bovine serum, glioblastoma 4 (GBM4) and glioblastoma 8 (GBM8) human tumor neurospheres were cultured in human basal neural stem cell medium: NeuroCult ${ }^{\mathbb{R}}$ NS-A Basal Medium (Human) supplemented with NeuroCult NS-A Proliferation Supplement (Human), epidermal growth factor, and basic fibroblast growth factor (obtained from Stem Cell Technologies Inc., Vancouver, BC, Canada). ${ }^{30}$ Dioleoylphosphatidylethanolamine (DOPE), cholesterol, distearoylphosphatidylcholine (DSPC), and 1,2-distearoylsn-glycero-3-phosphothanolamine-B-[methoxy(polyethylene glycol)-2000] (DSPE-mPEG2000) were purchased from Avanti Polar Lipids (Alabaster, AL, USA). STS was purchased from LC Laboratories (Woburn, MA, USA). A Sephadex G-50 column was sourced from GE Healthcare (Little Chalfont, UK), and the Sepharose CL6B and Dulbecco's Modified Eagle's Medium were purchased from Sigma-Aldrich ( $\mathrm{St}$ Louis, MO, USA).

\section{Preparation of liposomes}

The composition of the liposomes included DSPC and was selected for stability and good payload retention, and PEG was attached to the liposomal surface to prevent opsonization and removal from the circulation by liver macrophages. The liposome formulation included cholesterol, DOPE, DSPC, and DSPE-mPEG2000 (6:6:6:1 molar ratio) in chloroform, and was evaporated under argon gas. ${ }^{31}$ The dried lipid film was hydrated with a buffer containing ammonium phosphate, ammonium sulfate, sodium phosphate, or sodium sulfate (300 mM, and either $\mathrm{pH} 3$ or $\mathrm{pH}$ 7.4), and vortexed for 1 minute to remove any adhering lipid film. Sonication in a bath sonicator (ULTRAsonik, Branson, Milford, CT, USA) for 1 minute at room temperature produced multilamellar vesicles. The multilamellar vesicles were in turn sonicated with a Ti-probe (450 sonicator; Branson Ultrasonics, Danbury, CT, USA) for 2 minutes to produce small unilamellar 
vesicles, indicated by formation of a translucent solution. Extrusion through a $100 \mathrm{~nm}$ pore size polycarbonate filter (Whatman, Maidstone, UK) was the final stage of a stepwise series of extrusions to reduce the size of the small unilamellar vesicles. A 6-(((4,4-difluoro-5-(2-thienyl)-4-bora-3a,4adiazas-indacene-3-yl)styryloxy)acetyl) aminohexanoic acid, succinimidyl ester (BODIPY) 630/650 fluorophore was conjugated to DOPE, and this fluorescent lipid was incorporated into the particle at low concentration for imaging the liposomes in vitro and in vivo.

\section{Reverse $\mathrm{pH}$ gradient for drug encapsulation}

STS at neutral and basic $\mathrm{pH}$ is in the free base form (solubility in water $10-20 \mu \mathrm{M})$ while in acidic medium $(10 \%$ citric acid) it forms an amine salt. We achieved a $10 \mathrm{mM}$ STS concentration. The prepared liposomes with internal buffer at $\mathrm{pH} 3$ or 7.4 were passed through a Sephadex G-50 column equilibrated with 4-(2-hydroxyethyl)-1-piperazineethanesulfonic acid (HEPES) buffered saline (20 mM HEPES, $150 \mathrm{mM}$ $\mathrm{NaCl}, \mathrm{pH}$ 7.4) or phosphate-buffered saline to exchange the external buffer. After buffer exchange, STS in 10\% citric acid at $\mathrm{pH} 3$ was added to the liposome suspension, or the $\mathrm{pH}$ of the liposome suspension was increased to 7.4 by adding $1 \mathrm{~N}$ sodium hydroxide. The $\mathrm{pH}$ was measured with a $\mathrm{pH}$ meter. The solution was heated at $50^{\circ} \mathrm{C}$ for 20 minutes, left to stand for 4 hours at room temperature, and then applied to a Sephadex G-50 column and eluted with MilliQ water (EMD Millipore Bedford, MA, USA).

\section{STS encapsulation efficiency}

Encapsulation efficiency was quantified using a direct method, measuring the STS absorbance of loaded liposomes, and by high-performance liquid chromatography (HPLC). The two methods yielded very similar results. Using the direct method, STS absorbance at $294 \mathrm{~nm}$ was obtained by subtracting the absorbance of empty liposomes from that of STS-loaded liposomes. The concentration of encapsulated STS was then interpolated from a standard curve of absorbance versus concentration. Encapsulation efficiency was calculated by dividing the amount of STS entrapped by the total amount used to load the liposomes, multiplied by 100 . For HPLC-based determination of encapsulation efficiency, the liposomes were first lyophilized and dissolved in HPLC grade methanol. The evaporation residue was dissolved in 100\% HPLC grade acetonitrile, and reverse phase HPLC was performed using an Agilent 1100 series HPLC instrument (Agilent, Santa Clara, CA, USA) with an ultraviolet detector. Samples were injected into a Zorbax C18 column (Agilent) with an injection volume of $50 \mu \mathrm{L}$. Elution was performed with a mobile phase composed of $80 \%$ acetonitrile, $10 \%$ methanol, and $10 \%$ water $(0.1 \%$ trifluoroacetic acid $)$ at a flow rate of $0.8 \mathrm{~mL}$ per minute. STS was detected using ultraviolet light at $294 \mathrm{~nm}$ and $20^{\circ} \mathrm{C}$. The concentration of STS was interpolated from a standard curve, and multiplied by 100 to yield the encapsulation efficiency of STS.

The published limit of detection of STS using liquid chromatography tandem mass-spectrometry is approximately 5 pmol, but more practically with ultraviolet detection the limit of detection value for STS is in the low ng range $(20 \mathrm{ng} / \mathrm{mL})$, while the limit of quantification in plasma using HPLC with ultraviolet detection is approximately $0.1 \mu \mathrm{g} / \mathrm{mL} .^{32,33}$

\section{In vitro STS retention studies}

Drug payload retention within liposomes is an important parameter that relates to circulation time and enhanced tumor accumulation of drug-loaded liposomes. ${ }^{34}$ Hence we sought to measure retention of STS. STS-loaded liposomes were incubated in phosphate-buffered saline or $100 \%$ human serum at room temperature, and the experiment was repeated twice. Deidentified human serum was obtained via the UC San Diego Moores Cancer Center from human subjects providing informed consent (institutional review board approval number 100936). The STS concentration remaining in the liposomes was determined at $0.5,1,2,4,6,12,24$, and 48 hours of incubation in phosphate-buffered saline or serum. For retention studies in phosphate-buffered saline, we used ultraviolet detection, and for plasma we used HPLC with ultraviolet detection. Separation of the liposomes from both phosphate-buffered saline and serum was performed by size exclusion chromatography. The phosphate-buffered saline liposome suspension was applied to a Sephadex G-50 column, while a Sepharose CL6B column was used for serum, both eluted with phosphate-buffered saline. ${ }^{35}$ After separation, the concentration of STS within the liposomes incubated in phosphate-buffered saline was determined spectrophotometrically at $294 \mathrm{~nm}$. The liposomes separated from serum were lyophilized and dissolved in methanol, and HPLC was performed as described earlier.

\section{Activity of encapsulated STS against human glioblastoma cells in vitro}

We evaluated the cytotoxic effect of STS-encapsulating liposomes and free STS on established human cancer cell lines, including A172, U251, U118, and U87 glioblastoma cells, and PC3 pancreatic and MDA-MB-231 breast cancer cells. All cells 
were grown in 96-well plates in complete medium with $10 \%$ fetal bovine serum at $37^{\circ} \mathrm{C}$. We also used established patientderived glioblastoma cell lines in the form of cancer stem cells acquired from fresh surgical isolates and grown in NeuroCult at $37^{\circ} \mathrm{C} .{ }^{36}$ With all cell lines, either free or liposomal STS was added and the cells were incubated for 72 hours. Tumor cell viability was evaluated using either the 3-(4,5-dimethylthiazol2-yl)-2,5-diphenyltetrazolium bromide (MTT) (Sigma-Aldrich) or Alamar blue assays (AbD Serotec, Raleigh, NC, USA). The amount of STS loaded for in vitro testing and cell viability was the maximum that we could load. This was because our goal was to maximize the quantity of STS loaded into the liposomes so that tumor delivery would be enhanced to the greatest degree possible.

For experiments with free STS, $10 \mathrm{mmol} / \mathrm{L}$ stocks were first serially diluted in dimethylsulfoxide then with medium, to avoid precipitation. The concentration of free and liposomal STS added to cultures ranged between $200 \mathrm{~nm}$ and $0.1 \mathrm{~nm}$, with two-fold decrements down the range. For both free and liposomal STS, cell viability was quantified using the MTT assay in all lines except GBM4 and GBM8. ${ }^{37}$ The absorbance at $540 \mathrm{~nm}$ was measured after adding MTT. Results were expressed as: percent viability $=($ Absorbance $(A) 540$ [treated cells $]-$ background $/ A 540$ [untreated cells] - background) $\times 100 \%$. In GBM4 and GBM8 lines, cell viability was determined by adding Alamar blue to the cells and 6-12 hours later measuring fluorescence using excitation and emission wavelengths of $560 \mathrm{~nm}$ and $590 \mathrm{~nm}$, respectively. ${ }^{38}$ Results were expressed as: percent viability $=(F 1[$ treated cells $]-$ background $/ F[$ untreated cells] - background) $\times 100 \%$. Dose-response curves were plotted using GraphPad ${ }^{\circledR}$ Prism software (Graphpad Software, Inc., La Jolla, CA, USA) and inhibitory concentration 50\% $\left(\mathrm{IC}_{50}\right)$ values were calculated.

Fluorescence-activated cell sorting (FACS; BD Biosciences, Franklin Lakes, NJ, USA) analysis for apoptosis was performed to verify that both free and liposomal STS induced apoptosis compared with vehicle controls. Human U87 GBM cells were plated in a six-well plate at a density of 200,000 cells per well. After 18 hours of plating, the cells were incubated for 32 hours with either $200 \mathrm{nM}$ of free STS, liposome-encapsulated STS, empty liposomes, or dimethylsulfoxide. This experiment was performed twice. The cells were stained using propidium iodide, which enters dead or dying cells, but cannot in a clear-cut way discriminate between primary necrosis and apoptosis. The cell population was measured by FACS, and the acquired data were analyzed using NIH Image J software (freeware from National Institutes of Health, Bethesda, MD, USA). Cell death was quantified by measuring the proportion of cells with fragmented DNA, ie, the sub $G_{1}$ fraction ( $G_{0}$ fraction).

\section{Mechanism of glioblastoma cell killing in vitro}

Western blot analysis was performed for Akt, phospho-Akt, and cleaved PARP as an index of apoptosis. U87 cells were placed in a six-well plate at a density of 200,000 cells per well. After 18 hours of plating, the cells were incubated with $200 \mathrm{nM}, 10 \mathrm{nM}$, or $0.1 \mathrm{nM}$ of STS encapsulated in liposomes, free STS, or the control for 32 hours, and lysed with NP40 lysis buffer containing protease inhibitor (cOmplete, Mini; Roche Applied Sciences, Indianapolis, IN, USA) and phosphatase inhibitor cocktail (Sigma-Aldrich). The protein concentration was determined using a Bio-Rad protein assay kit (Bio-Rad Laboratories, Hercules, CA, USA). Equal amounts of protein $(40 \mu \mathrm{g})$ were resolved on $4 \%-12 \%$ Nupage $^{\circledR}$ Bis-Tris polyacrylamide gels (Invitrogen, Carlsbad, CA, USA). Protein was transferred to a nitrocellulose membrane and probed with primary antibodies to Akt, phospho-Akt, or cleaved PARP (Cell Signaling Technologies Inc., Danvers, MA, USA). Bands were detected by chemiluminescence (Amersham Pharmacia Biotech, Piscataway, NJ, USA).

\section{Biodistribution of liposomes}

Mice were subcutaneously injected with $2 \times 10^{6}$ U87 cells. After the tumors reached $40-50 \mathrm{~mm}^{3}$, the mice were injected with BODIPY fluorescent dye-labeled liposomes containing STS. Four hours after the initial injection, fluorescein isothiocyanate-lectin was injected to label the tumor vascular endothelium. The tumor, liver, kidney, heart, and lung tissues were removed, sectioned, and examined under a scanning confocal microscope (Nikon Corporation, Tokyo, Japan). We acquired five images per organ and the BODIPY pixel count was normalized by the total pixels in each acquisition, which was the same size for all organ image acquisitions. BODIPY is covalently conjugated to the lipid and present within the liposomal bilayer, so was unlikely to be liberated. The labeled pixels were counted and normalized against the total pixels of each image.

\section{In vivo antitumor activity and systemic toxicity of encapsulated STS}

Athymic nu/nu mice aged 5-6 weeks were subcutaneously inoculated in both the right and left flank with 2 million U87 glioblastoma cells. In one group ( $n=4$; total of eight tumors), the tumors were allowed to grow very large $\left(180-200 \mathrm{~mm}^{3}\right)$, while in a second group $(\mathrm{n}=5$; total of ten 
tumors), the tumors were allowed to establish but the experiment was started earlier, when they reached $40-50 \mathrm{~mm}^{3}$. The mice in both tumor volume groups were sorted so that the treatment and control groups had the same average tumor size.

For the initial experiment, mice bearing tumors allowed to reach 180-200 $\mathrm{mm}^{3}$ were treated with three intravenous doses per week for 2 weeks of either phosphate-buffered saline, free STS $(0.8 \mathrm{mg} / \mathrm{kg})$, or encapsulated STS $(0.8 \mathrm{mg} / \mathrm{kg})$. The second cohort with $40-50 \mathrm{~mm}^{3}$ tumors was injected with either $200 \mu \mathrm{L}$ of encapsulated STS for a $0.8 \mathrm{mg} / \mathrm{kg}$ STS dose three times per week for 3 weeks, or with $200 \mu \mathrm{L}$ of empty liposomes or phosphate-buffered saline according to the same time schedule.

In all study groups, the tumors were measured weekly using calipers and volume was calculated using the standard formula: $\mathrm{V}=\left(\right.$ length $\times\left[\right.$ width $\left.\left.^{2}\right]\right) / 2 .{ }^{39}$ The mice were sacrificed when the tumors reached $1,500 \mathrm{~mm}^{3}$, in accordance with our Institutional Animal Care and Use Committee protocol. Studies show that free STS clears from the circulation immediately, and the elevated doses required to bypass this effect are acutely toxic, while lower doses may have longer-term toxicity. ${ }^{40,41}$ In order to determine whether liposomal encapsulation of STS reduced toxicity, body weight was measured before and after the treatment period, because loss of body weight is a generally reliable sign of toxicity seen after STS treatment, and is often used as a toxicity measure in murine studies of cancer chemotherapy agents. ${ }^{42-44}$

Tumor cell proliferation with liposomal STS was assessed using Ki-67 staining. Paraffin-embedded tumor xenografts from animals treated with phosphate-buffered saline, free STS, and liposomal STS were sectioned, deparaffinized, heated at $97^{\circ} \mathrm{C}$ for 30 minutes for antigen retrieval, and blocked with peroxidase, $2.5 \%$ horse serum, and avidin/biotin. Sections were incubated with Ki-67 antibody (Abcam, Cambridge, MA, USA) for one hour at room temperature and then with secondary antibody for 30 minutes at room temperature. Slices were stained with DAB peroxidase substrate solution (Vector Laboratories, Burlingame, CA, USA) and counterstained with hematoxylin. The extent of Ki-67 staining was tabulated by counting stained cells at $40 \times$ field using a light microscope (Nikon Corporation). Three separate fields of each slide were counted for Ki-67-positive cells.

\section{Statistical analysis}

All statistical analyses were performed with Excel (Microsoft, Redmond, WA, USA) or Prism (GraphPad). The statistical significance of the experiments was determined by a twotailed Student's $t$-test (parametric) and Mann-Whitney $U$ test (nonparametric). $P$-values $<0.05$ were considered to be statistically significant. Both parametric and nonparametric tests were applied to address the possibility that the group variances may have differed. In all figures, the vertical bars represent the mean value \pm standard deviation. In order to compare tumors, $t$-tests were performed at the final time point.

\section{Results}

\section{Physical characterization of liposomes}

Liposomal size and charge was measured with the STS-loaded liposomes in water, in phosphate-buffered saline, and in $10 \mathrm{mM} \mathrm{NaCl}$ (Supplementary Table 1). The average liposomal size in distilled water was $108 \pm 1.1 \mathrm{~nm}$, which was very close to that in phosphate-buffered saline or $\mathrm{NaCl}$ (three measurements each, Supplementary Table 1). The zeta potential or liposome surface charge in all types of medium was close to the desired neutrality (Supplementary Table 1, ten measurements in each medium). Scanning electron microscopy data confirmed that the STS-loaded liposomes in distilled water were spherical and intact (Supplementary Figure 1A). The polydispersity index was low in water, in phosphate-buffered saline, and in $10 \mathrm{mM} \mathrm{NaCl}$, at $0.15,0.25$, and 0.25 . This is consistent with a homogeneous size distribution and absence of aggregation.

\section{Encapsulation efficiency Reverse $\mathrm{pH}$ gradient with ammonium-based buffers produced effective encapsulation}

Liposomes were prepared by hydrating the lipid film with ammonium and sodium buffers. The extraliposomal buffer was exchanged through different external buffers (HEPES, phosphate-buffered saline) and purification achieved by size exclusion chromatography (Figure 1). Figure 1A shows the effect of internal buffer composition and Figure 1B shows the effect of internal buffer $\mathrm{pH}$. For these experiments, the external buffer $\mathrm{pH}$ was kept constant. The best encapsulation efficiencies of $70 \%$ and $65 \%$ were achieved when the internal buffer was ammonium phosphate or ammonium sulfate, respectively, and $\mathrm{pH}$ was 7.4 (Figure 1A and B). Sodium phosphate and sodium sulfate buffers produced very low encapsulation of $3 \%-4 \%$. The encapsulation efficiency was the same whether the milieu external to the liposomes consisted of HEPES or phosphate-buffered saline (Figure 1C). 

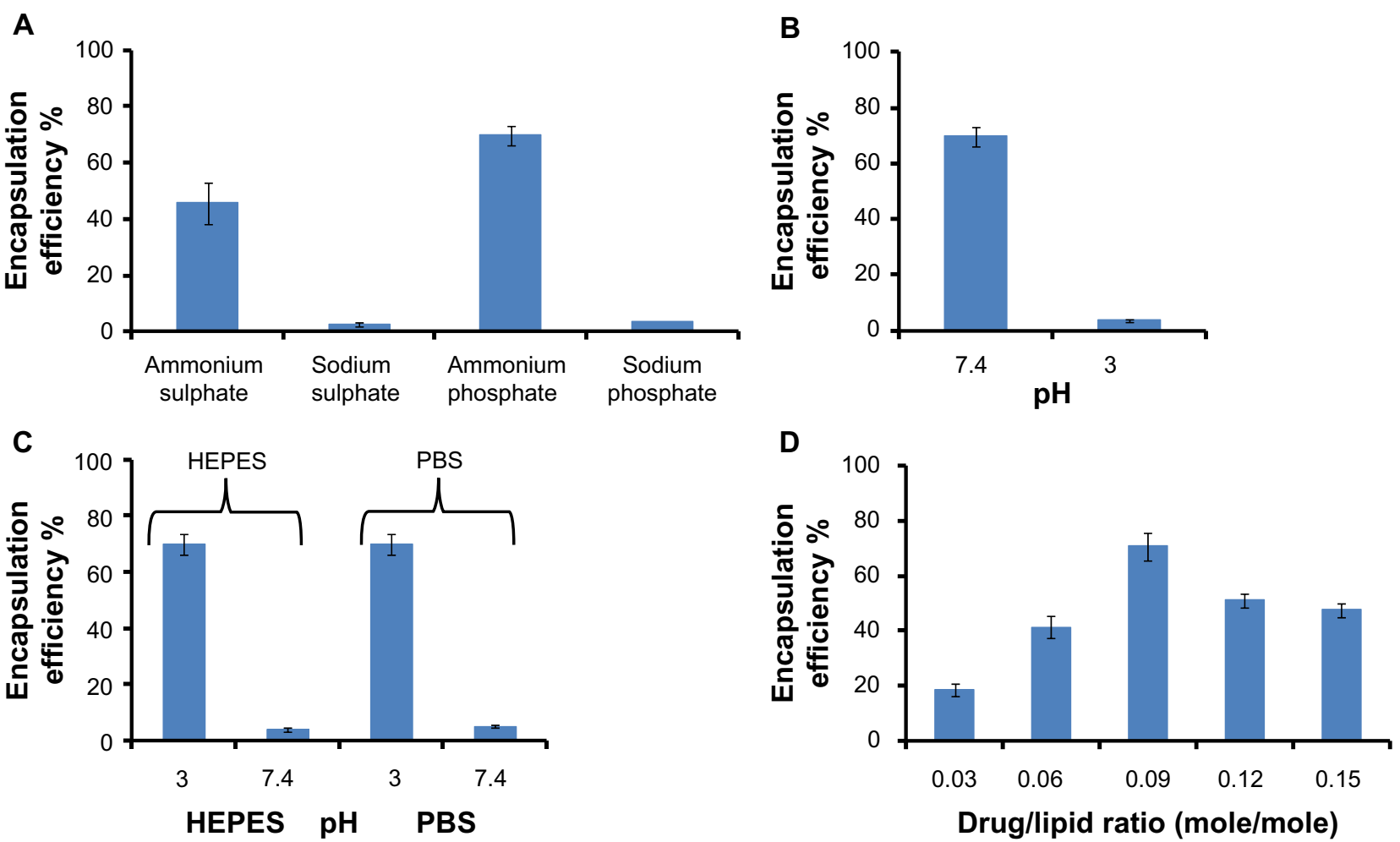

Figure I Parameters affecting staurosporine encapsulation in liposomes - the importance of a reverse pH gradient, buffer composition, and drug-to-lipid ratio. Each experiment for all four panels was repeated three times. (A) Encapsulation of staurosporine in liposomes driven by different internal buffers ( $\mathrm{n}=3$ ). (B) Effect of internal buffer $\mathrm{pH}$ on staurosporine encapsulation. The external buffer was held at constant $\mathrm{pH}$ 3. (C) Effect of external buffer and its $\mathrm{pH}$. The internal buffer was held at constant $\mathrm{pH}$ 7.4. (D) Encapsulation efficiencies of liposomal staurosporine at increasing initial drug-to-lipid ratios (mole/mole). Each data point represents mean encapsulation efficiency calculated from three samples.

Abbreviations: PBS, phosphate-buffered saline; HEPES, 4-(2-hydroxyethyl)-I-piperazineethanesulfonic acid.

\section{Optimal drug-to-lipid ratio for efficient}

\section{STS encapsulation}

STS was added in drug-to-lipid ratios of $0.03,0.06,0.09$, 0.12 , or 0.15 (mole/mole). STS uptake into the liposomes was measured as described. Liposomal loading capacity was highest when the drug-to-lipid ratio was 0.09 (mole/mole), with a peak value of $70 \%$, compared with $25 \%$ obtained for previously described formulations (Figure 1D). ${ }^{28}$ Our spectrophotometric measurements indicated that we loaded $81.5 \mu \mathrm{g}$ of STS for each $1 \mathrm{~mL}$ of liposomes. The optimal loading regime including the reverse $\mathrm{pH}$ gradient, ammonium phosphate buffer, and optimal drug-to-lipid ratio was used for all the subsequent in vitro/vivo experiments in this study.

\section{In vitro liposomal retention of STS}

Liposomal retention ${ }^{34}$ studies performed in duplicate revealed that the encapsulating liposomes were stable for several hours, with comparatively little leakage of payload. Figure $2 \mathrm{~A}$ and B reveal that after 2 hours of incubation in human serum, respectively, the liposomes retained more than $96 \%$ of the initial load of STS. The liposomes were not formulated to be thermosensitive and although we did not test at $37^{\circ} \mathrm{C}$, we selected our lipids for stability and short-term ( $0-6$ hours) payload retention within the temperature range of $22^{\circ} \mathrm{C}$ to $37^{\circ} \mathrm{C} . .^{45} \mathrm{We}$ used DSPC lipid, which has a phase transition temperature of approximately $55^{\circ} \mathrm{C}$, and DSPC liposomes have demonstrated stable payload retention between $4^{\circ} \mathrm{C}$ and $37^{\circ} \mathrm{C} .{ }^{46}$ By 6 hours of incubation in serum, the liposomes retained $82 \%$ of the STS, $55 \%$ after 12 hours, $25 \%$ after 24 hours, and $5 \%$ after 48 hours. In phosphate-buffered saline, $90 \%$ of the drug was retained after 4 hours, $56 \%$ after 6 hours, $45 \%$ after 12 hours, $42 \%$ after 24 hours, and $23 \%$ after 48 hours (Figure 2).

\section{Activity of encapsulated STS against glioblastoma cells in vitro}

Encapsulation of STS within liposomes did not impede its cytotoxic effect. We evaluated the inhibitory effect of free and encapsulated STS on established and freshly derived human solid tumor cell lines by measuring cell viability (Figure 3A, Supplementary Figure $2 \mathrm{~A}-\mathrm{G}$ ). The results with both patientderived stem cells and established brain tumor lines show 


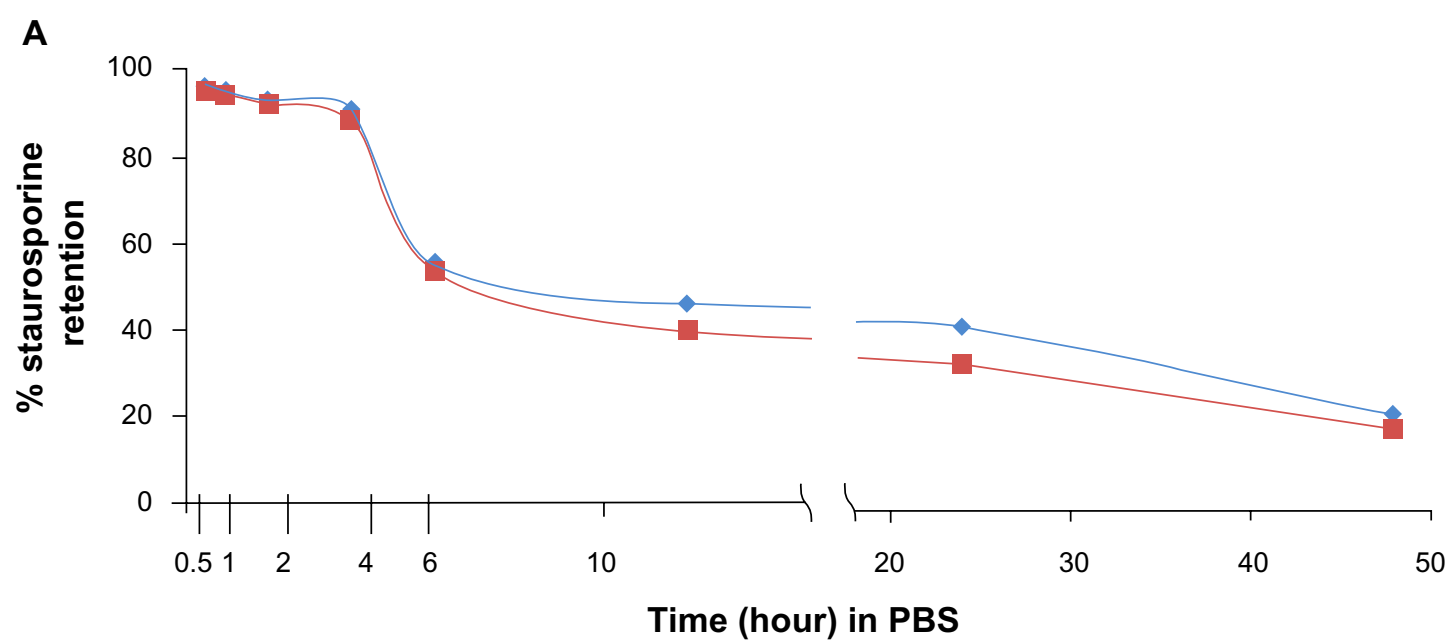

B

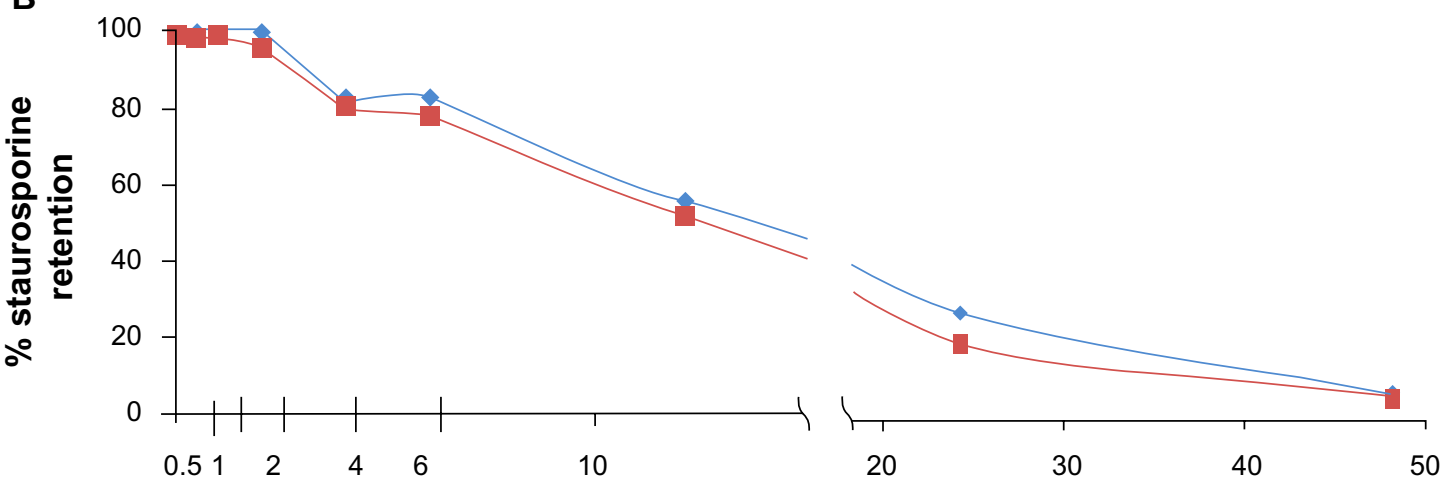

Time (hour) in $100 \%$ human serum

Figure 2 Gradient-loaded liposomes are stable with good payload retention in vitro. Liposomes suspended in (A) PBS and (B) human serum. Graphs show results of duplicate tests. Retention was measured after incubation in PBS/serum at room temperature for a range of times. Virtually I00\% staurosporine was contained within the liposomes by 3 hours of incubation in PBS or serum, with approximately $80 \%$ of the staurosporine remaining after 6 hours of incubation. Red $=$ expt I; blue $=$ expt 2 .

Abbreviation: PBS, phosphate-buffered saline; expt, experiment.

that the $\mathrm{EC}_{50}$ of free and liposomal STS was comparable (Supplementary Figure 2). Potency in these cell lines, and in the PC3 prostate tumor line, ranged from approximately $1 \mathrm{nM}$ to $13 \mathrm{nM}$. The $\mathrm{EC}_{50}$ for the MDA-MB-231 breast tumor line was higher, at $113 \mathrm{nM}$ with liposomal STS, but better than free STS with an $\mathrm{EC}_{50}$ of $507 \mathrm{nM}$. In addition, the FACS results indicated that both free and liposomal STS induced cell death compared with the controls, which were empty liposomes alone and dimethylsulfoxide alone (Figure 3B). The $\mathrm{G}_{0}$ fraction increased markedly from approximately $2.4 \%$ in controls to $54 \%$ with free STS and $62.9 \%$ with liposomal STS.

\section{Mechanism of STS-induced glioblastoma cell killing in vitro}

Morphologic changes clearly indicated a direct toxic effect on the cells, which rounded up and assumed an appearance consistent with apoptosis (Supplementary Figure 3). The cells showed evidence that fluorescent liposomes entered the cells (Supplementary Figure 3). The Western blot results for PARP cleavage were consistent with induction of apoptosis (Figure 3C). The Western blot analysis also indicated that Akt phosphorylation was blocked by STS (Figure 3C). ${ }^{47,48}$ In addition, we tested free and liposomal STS with other cell lines, including A172, U118, U251, GBM4, GBM8, PC3, and MDA-MB-231. All cell lines were driven into apoptosis and exhibited similar $\mathrm{IC}_{50}$ values with the free compound and the liposomal compound.

\section{Biodistribution of liposomes}

Figure 4A depicts liposomal accumulation within the sectioned U87 tumors growing subcutaneously in the mouse flank. Figure $4 \mathrm{~B}$ is the quantitation for each listed organ from which five slices were acquired and imaged for two mice. The highest relative density of fluorescently labeled liposomes was recorded in the tumor tissue, although accumulation of the label was 


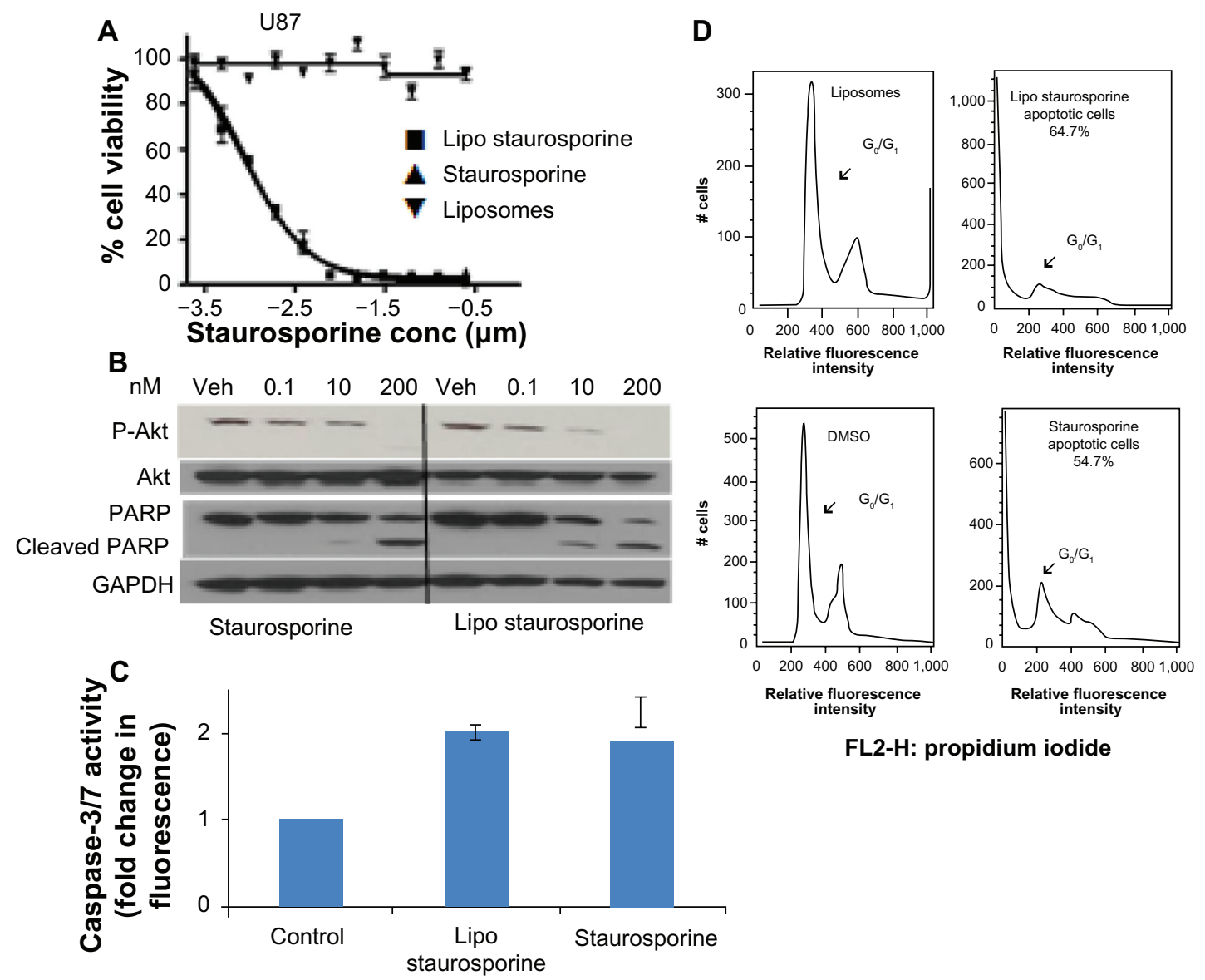

Figure 3 Potency and mechanism of liposomal staurosporine in vitro. (A) In vitro MTT cell viability assay results for free and liposomal staurosporine versus empty liposomes (control) were determined with U87 glioblastoma cells. First, 2,000 cells were plated and after 18 hours free or liposomal staurosporine was added. The concentration of free or liposomal staurosporine added to cultures ranged between $200 \mathrm{~nm}$ and $0.1 \mathrm{~nm}$, with two-fold decrements down the range. Free and liposomal staurosporine had very similar potency while empty liposomes caused essentially no tumor cell killing. (B) Western blot analysis for Akt, P-Akt, and cleaved poly(ADP-ribose) polymerase. U87 cells were incubated with $200 \mathrm{nM}, 10 \mathrm{nM}$, and $0.1 \mathrm{nM}$ of staurosporine encapsulated in liposomes and staurosporine for 32 hours. (C) Bar graph showing caspase-3/7 activity as fold change from control (which is I). Free and liposomal staurosporine yielded similar changes in caspase activity. (D) Fluorescence-activated cell sorting analysis for U87 glioblastoma cells incubated with either empty liposomes, $200 \mathrm{nM}$ of staurosporine encapsulated in liposomes, dimethylsulfoxide, or free staurosporine. Representative plots of two repeats per condition are shown, and reveal that both free and liposomal staurosporine induced cell death, indicated by a $\mathrm{G}_{0} / \mathrm{G}_{1}$ shift, compared with the dimethylsulfoxide and empty liposome control treatments.

Abbreviations: conc, concentration; MTT, (3-(4,5-dimethylthiazol-2-yl)-2,5-diphenyltetrazolium bromide; Lipo, liposomal; PARP, poly(ADP-ribose) polymerase; GADPH, Glyceraldehyde 3-phosphate dehydrogenase; Akt, protein kinase B; DMSO, Dimethyl sulfoxide; Veh, DMSO vehicle control.
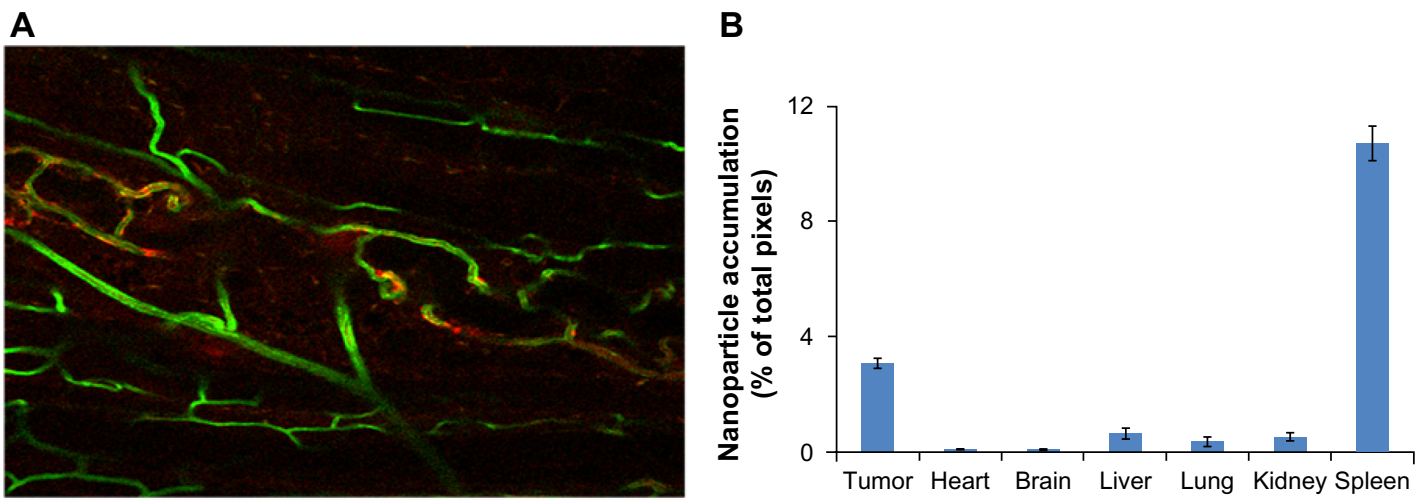

Figure 4 Liposomal BODIPY (boron-dipyrromethene) fluorescence attained a relatively high density in tumor tissue. (A) Liposomes (labeled red) clearly entered the tumor and accumulated at tumor microvessels (labeled green). ( $20 \times$ scanning confocal image). (B) The bar graph shows the liposomal BODIPY pixel density for two mice bearing tumors that were injected intravenously with liposomes. Five images were acquired per organ in two mice, the pixel density was normalized by the total number of acquired pixels, and all organs were imaged with the same magnification. As expected, the spleen showed a high accumulation, but normal brain tissue showed very low accumulation. The filled columns indicate the average value while the superimposed line bars delineate the range. 
seen in other organs, in particular the spleen. It is noteworthy that very little accumulation was seen in the normal brain. The data do not reveal absolute amounts, but are nonetheless encouraging and consistent with an improvement of pharmacokinetics and biodistribution, providing a basis for subsequent, comprehensive biodistribution studies incorporating fluorescence and liquid chromatography-mass spectroscopy.

\section{Encapsulated STS exhibits in vivo antitumor activity with no evidence of toxicity based on body weight}

We found that a relatively high dose of both free STS and liposomal STS (5 mg/kg) was acutely lethal, but $0.8 \mathrm{mg} / \mathrm{kg}$ was not, and the equivalent in vitro dose $(40 \mathrm{nM})$ attained $100 \%$ tumor cell killing. Based on the literature, a $0.8 \mathrm{mg} /$ $\mathrm{kg}$ dose injected as free STS in rodents would be entirely cleared after only a few circulatory passes, and no demonstrable effect on tumor growth would even be feasible. ${ }^{33}$

When the tumors were allowed to grow very large $\left(>180 \mathrm{~mm}^{3}\right)$ before treatment was started, treatment with liposomal STS over 2 weeks markedly slowed the growth of the tumors relative to those treated with phosphatebuffered saline and free STS. The plots in Figure 5A reveal a substantial and statistically significant effect (parametric $P<0.0004$; nonparametric $P=0.00018$ ), and the photographs in Figure 5B suggest little apparent variation in size. Free STS had no demonstrable antitumor effect and was toxic, as body weight did decline (parametric $P=0.0003$; nonparametric $P=0.005$ ), while animals treated with liposomal STS or phosphate-buffered saline had no weight difference (Figure 5C). Ki-67 staining in tumor tissue revealed that tumor proliferation was significantly reduced with liposomal STS (parametric and nonparametric, $P<0.03$; Figure 5D).

We next examined the antitumor effect when treatment was started with smaller $\left(40-50 \mathrm{~mm}^{3}\right)$ but well established and rapidly growing tumors. We compared tumor growth in mice that were treated with empty liposomes versus those treated with liposomal STS. Figure $6 \mathrm{~A}$ and B reveal complete suppression of tumor growth with liposomal STS at $0.8 \mathrm{mg} / \mathrm{kg}$ (parametric $P<0.0006$; nonparametric $P=0.00044$ ).

Body weight did not differ between the liposomal STS and empty liposome cohorts in the small tumor $\left(40-50 \mathrm{~mm}^{3}\right)$

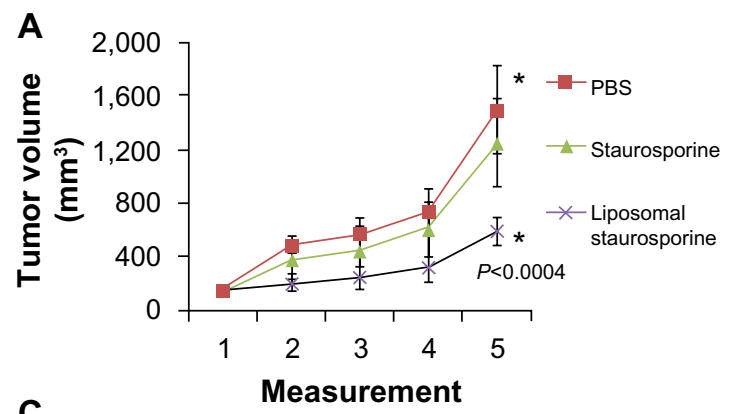

B

C

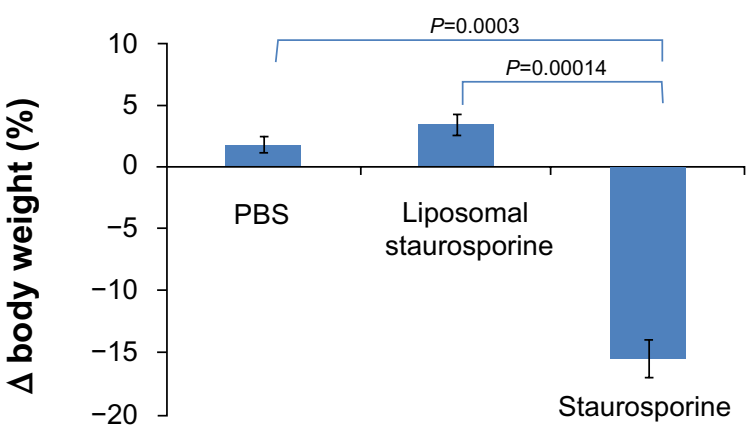

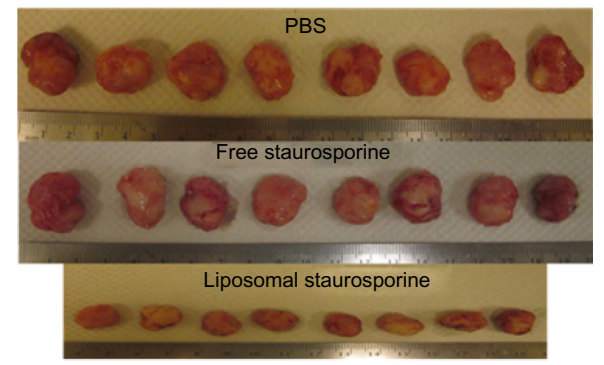

D

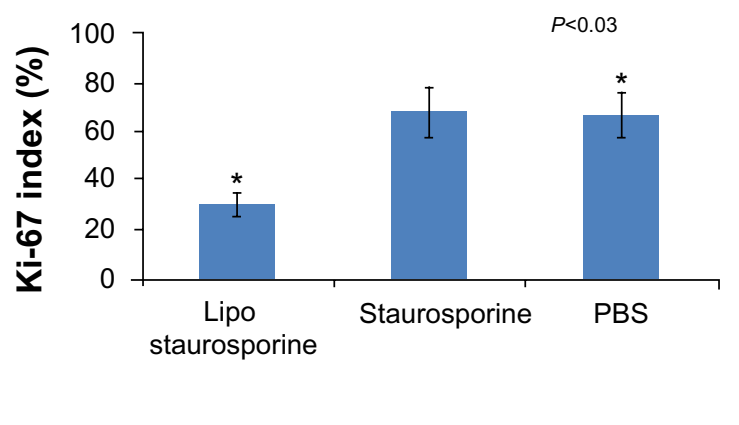

Figure 5 Liposomal staurosporine markedly suppressed $180-200 \mathrm{~mm}^{3}$ U87 flank tumors with no body weight-related evidence of toxicity. There were four subjects per group for tumor size, tumor images, body weight, and Ki-67. (A) Control $(\square)$, liposomal staurosporine $(X)$ and free staurosporine $(\triangle)$. The $x$-axis represents measurements (mean \pm standard deviation) on successive days after tumor implantation (Parametric $P<0.0004$, shown in figure; nonparametric $P=0.000$ I 8 ). (B) Tumor images for liposomal staurosporine, free staurosporine, and PBS groups. (C) Body weight bar graph showing a significant decline in body weight with free staurosporine, indicating toxicity (parametric $P=0.0003$, shown in figure; nonparametric $P=0.005$ ). Note for the liposomal staurosporine group that, at the end of the treatment period, body weight as compared with pretreatment weight did not differ from the same measure in controls. (D) Proliferating tumor cells identified using Ki-67 in PBS, staurosporine, and liposomal staurosporine treatment groups. The PBS and liposomal staurosporine groups differed significantly (Parametric, shown in the figure, and nonparametric $P<0.03$ ) while PBS versus free staurosporine did not. * indicates comparison between PBC and liposomal staurosporine.

Abbreviations: PBS, phosphate-buffered saline; lipo, liposomal. 


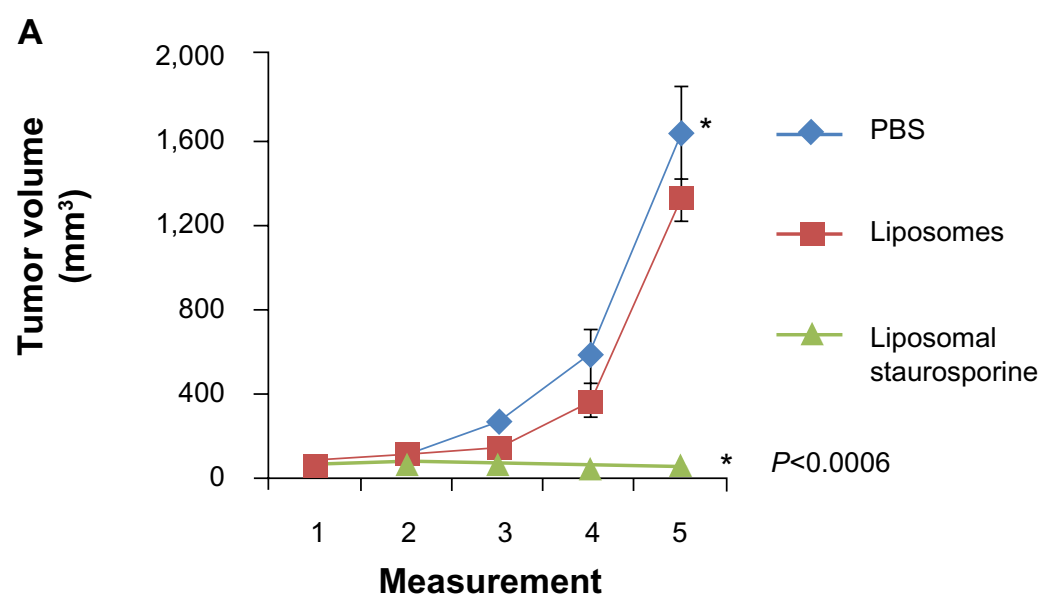

B

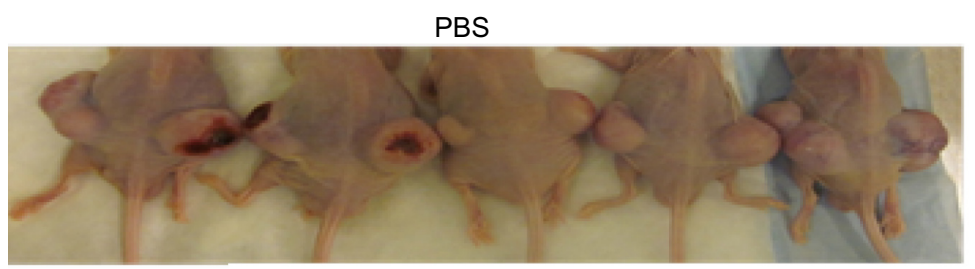

Liposomal staurosporine
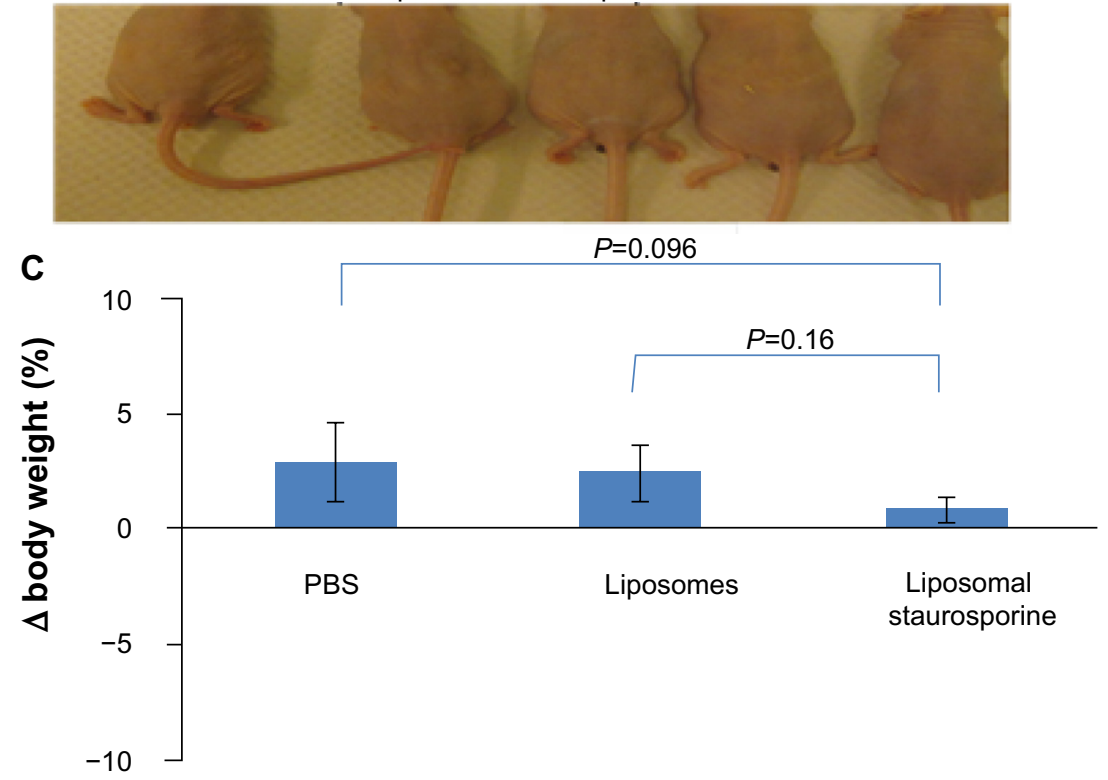

Figure 6 Liposomal staurosporine completely inhibited the growth of established $40-50 \mathrm{~mm}^{3}$ U87 flank tumors with no evidence of toxicity. PBS ( $)$ ), liposomes $(\square)$, liposomal staurosporine $(\triangle)$. The $x$-axis represents measurements on successive days after tumor implantation $(n=5$; parametric $P<0.0006$ shown in figure; nonparametric $P=0.00044)$. * indicates PBS versus liposomal staurosporine. (B) Photographs of nude mice implanted subcutaneously in both flanks with U87 cells and treated. (C) Body weights for mice treated with PBS, empty liposomes, or liposomal staurosporine. Unpaired, parametric and nonparametric comparisons indicated no significant difference between weights taken before versus after treatment ( $P B S$ versus liposomal staurosporine parametric $P=0.096$; empty liposomes versus liposomal staurosporine, parametric $P=0.16$ ). Abbreviation: PBS, phosphate-buffered saline.

group, again indicating a general absence of toxicity when STS was encapsulated (Figure 6C). Based on the literature, injection of $0.8 \mathrm{mg} / \mathrm{kg}$ STS in the free drug form is cleared from the circulation rapidly. Similarly, our results for the large tumor group (180-200 $\mathrm{mm}^{3}$ ) indicate that free STS at the doses we used had no detectable effect on tumor growth. ${ }^{8}$ We did not administer free STS to mice bearing the smaller $\left(40-50 \mathrm{~mm}^{3}\right)$ tumors because we found evidence of systemic toxicity in the large tumor group.

\section{Discussion}

Our study demonstrates inhibition of tumors established in vivo using liposomal STS administered at a low intravenous dose. Liposomal encapsulation reduced toxic- 
ity, as mice treated with liposomal STS experienced no loss of weight over the 41-day experimental period (Figures 5 and 6). In contrast, there was definite weight loss in mice injected with a comparable dose of free STS (Figure 5). Biodistribution data revealed that the liposomes concentrated in tumors (Figure 4B), most probably via the enhanced permeability retention effect, and targeting may further enhance selectivity. ${ }^{49}$ Notably, even when treatment was withheld until the tumors became much larger, liposomal STS markedly suppressed tumor growth (Figure 5). These are the first in vivo results for STS loaded into liposomes, and support the feasibility of further translational investigations of liposomal STS for cancer treatment.

Collectively it has been hypothesized that the pharmacokinetic and toxicity related limitations of STS could be overcome by liposomal encapsulation. ${ }^{50}$ The liposomes accumulated in the tumor and the spleen, as expected for a nanoparticle, and showed relatively much less accumulation in other organs. Interestingly, the normal brain has been found to have a low concentration which may be beneficial, assuming that liposomes accumulate in brain tumors via leakage from malformed tumor microvasculature, as demonstrated by Munson et al. ${ }^{51}$ We explored a gradient-based method owing to its simplicity and efficient loading. ${ }^{52,53}$ Earlier gradient loading studies reported that liposomal uptake of drugs was driven by neutral external $\mathrm{pH}$ and low internal $\mathrm{pH}$. We tested this and other $\mathrm{pH}$ profiles in an effort to optimize loading and, for the first time, describe enhancement of loading by reversing the gradient profile, so that external $\mathrm{pH}$ was low while internal $\mathrm{pH}$ was neutral (Figure 1). Our methodology may be amenable for enhancement of liposomally encapsulating other potentially useful drug chemotypes that are currently limited due to poor loading.

We found that ammonium-based buffers resulted in higher loading compared with sodium salt buffers, and the best results were acquired using ammonium phosphate buffer. The reason is unknown, but ammonium groups are weakly acidic and can be transformed to ammonia, a weak base which is more lipophilic and could readily pass into the lipid bilayer, potentially affecting its barrier characteristics. We plan to address in a subsequent study the physical basis for improved loading with ammonium salt-based buffers, and to confirm the final loaded location of the STS. Moreover, given its chemical structure and hydrophobicity, it is quite possible that STS may have partitioned in the lipid bilayer of the liposomes, and a systematic experimental confirmation of this will be a primary focus in a forthcoming study.
Prevention of premature drug payload release from circulating liposomes is an essential requirement to ensure adequate accumulation of the compound in tumors..$^{8,34}$ Previous gradient loading methods have only supported liposomal retention of weakly basic anthracyclines such as doxorubicin and to some extent the camptothecin analogs. ${ }^{54,55}$ The loading methodology and liposomal composition we describe resulted in high ( $\geq 96 \%$ ) STS retention at 2 hours in human serum, with $82 \%$ remaining at 6 hours (Figure 2). Thus, the liposomal bilayer did not require extensive modification or addition of stabilizers to slow payload efflux. Given that we observed liposomes accumulating at the tumor site by 5 hours after intravenous injection, the favorable liposomal retention of STS in serum would support effective tumor delivery. Future studies may examine the relationship with cellular retention of STS, because recent data indicate that this is crucial for STS-induced cell death in the model organism, Neurospora crassa. ${ }^{56}$

Liposomal encapsulation did not change the nature or mechanism of cell killing by STS when compared with the free drug (Figure 3). Moreover, the in vivo antitumor effect of liposomal STS was robust in the subcutaneous U87 tumor model, while the free compound did not elicit a detectable antitumor effect (Figures 5 and 6). The U87 model was used as a general platform since we aimed to explore the feasibility of liposomal STS as a general agent for resistant solid tumors (Supplementary Figure 2). In terms of brain tumors, a recent report has shown tumor accumulation via leaky tumor vasculature and a robust antitumor effect of liposomal doxorubicin in the rat C6 glioma. ${ }^{51}$ Owing to its high degree of potency, we expect that even a low concentration of STS in brain tumors may exert a useful therapeutic effect. In our system, liposomal STS completely suppressed 40-50 $\mathrm{mm}^{3}$ tumors, and even much larger tumors (in the order of $>180 \mathrm{~mm}^{3}$ ) had a significant reduction in growth, even with delayed treatment (Figures 5 and 6). The growth kinetics of the large versus small tumors differed, with the smaller tumors growing faster (Figures 5 and 6). Although the basis for this is not known, one possible explanation is that the larger tumors outstripped their blood perfusion, and potentially became internally hypoxic and necrotic so that a smaller proportion of their mass was actively proliferating.

The present study was primarily focused on demonstrating the feasibility of liposomal STS to justify further, more comprehensive efficacy, pharmacokinetic, and toxicity studies, and hence has limitations in terms of interpretation. For example, more detailed dynamic studies showing the change in liposomal biodistribution and STS concentration during 
the first 48 hours and diverse measures of toxicity will be pursued in follow-up research. Such indepth experiments were beyond the scope and intent of the present study. In addition, while the lipids we selected for our liposomal formulation are stable between room and body temperature (Figure 2), later more detailed retention optimization studies will be conducted at $37^{\circ} \mathrm{C}$ in vitro and will subsequently be validated by in vivo experiments.

In summary, this study demonstrated for the first time the antitumor activity of liposomal STS at a low systemic dose with no apparent toxicity. The compound was not cleared before a therapeutic effect could be induced, and biodistribution data revealed preferential tumor accumulation. Moreover, we developed an efficient method for liposomal STS encapsulation, which may also be effective for other drug chemotypes. The present report provides the basis for pursuing comprehensive preclinical studies on the efficacy, pharmacokinetics, biodistribution, and toxicology of a range of encapsulated STS doses for solid tumor therapy.

\section{Acknowledgments}

The authors wish to thank Santosh Aryal for technical assistance with the scanning electron microscopy imaging and HPLC, Sara Weis for technical assistance with confocal imaging, and Matthew Gallagher and Gabi Gomez for technical assistance in the laboratory. We also thank Lori Davis for her excellent editorial and proofreading assistance. This work was supported in part by grants from the National Institutes of Health (NIH 3P30CA023100-25S8) and the Tuttleman Family Foundation to SK, Department of Defense (Armey) IDEA Awared BC095376, NIH 5R21CA164BB0-02 to DS.

\section{Disclosure}

The authors report no conflicts of interest in this work.

\section{References}

1. Tamaoki T, Nomoto H, Takahashi I, Kato Y, Morimoto M, Tomita F. Staurosporine, a potent inhibitor of phospholipid/Ca++dependent protein kinase. Biochem Biophys Res Commun. 1986;135(2): 397-402.

2. Zhang XD, Gillespie SK, Hersey P. Staurosporine induces apoptosis of melanoma by both caspase-dependent and -independent apoptotic pathways. Mol Cancer Ther. 2004;3(2):187-197.

3. Schwartz GK, Redwood SM, Ohnuma T, Holland JF, Droller MJ, Liu BC. Inhibition of invasion of invasive human bladder carcinoma cells by protein kinase C inhibitor staurosporine. J Natl Cancer Inst. 1990;82(22):1753-1756.

4. Tang D, Lahti JM, Kidd VJ. Caspase-8 activation and bid cleavage contribute to MCF7 cellular execution in a caspase-3-dependent manner during staurosporine-mediated apoptosis. J Biol Chem. 2000;275(13): 9303-9307.
5. Baltuch GH, Dooley NP, Couldwell WT, Yong VW. Staurosporine differentially inhibits glioma versus non-glioma cell lines. J Neurooncol. 1993;16(2):141-147.

6. Fuse E, Tanii H, Kurata N, et al. Unpredicted clinical pharmacology of UCN-01 caused by specific binding to human alpha1-acid glycoprotein. Cancer Res. 1998;58(15):3248-3253.

7. Akinaga S, Gomi K, Morimoto M, Tamaoki T, Okabe M. Antitumor activity of UCN-01, a selective inhibitor of protein kinase C, in murine and human tumor models. Cancer Res. 1991;51(18):4888-4892.

8. Gurley LR, Umbarger KO, Kim JM, Bradbury EM, Lehnert BE. Staurosporine Analysis and its Pharmacokinetics in the Blood of Rats. Los Alamos, NM: Los Alamos National Laboratory; 1994.

9. Gescher A. Analogs of staurosporine: potential anticancer drugs? Gen Pharmacol. 1998;31(5):721-728.

10. Jarvis WD, Grant S. Protein kinase C targeting in antineoplastic treatment strategies. Invest New Drugs. 1999;17(3):227-240.

11. Testa JR, Bellacosa A. AKT plays a central role in tumorigenesis. Proc Natl Acad Sci U S A. 2001;98(20):10983-10985.

12. Vivanco I, Sawyers CL. The phosphatidylinositol 3-Kinase AKT pathway in human cancer. Nat Rev Cancer. 2002;2(7):489-501.

13. Sato S, Fujita N, Tsuruo T. Interference with PDK1-Akt survival signaling pathway by UCN-01 (7-hydroxystaurosporine). Oncogene. 2002;21(11):1727-1738.

14. Baldwin RM, Parolin DA, Lorimer IA. Regulation of glioblastoma cell invasion by PKC iota and RhoB. Oncogene. 2008;27(25):3587-3595.

15. Lawrie AM, Noble ME, Tunnah P, Brown NR, Johnson LN, Endicott JA. Protein kinase inhibition by staurosporine revealed in details of the molecular interaction with CDK2. Nat Struct Biol. 1997;4(10):796-801.

16. Friedman B, Fujiki H, Rosner MR. Regulation of the epidermal growth factor receptor by growth-modulating agents: effects of staurosporine, a protein kinase inhibitor. Cancer Res. 1990;50(3):533-538.

17. da Rocha AB, Mans DR, Regner A, Schwartsmann G. Targeting protein kinase $C$ : new therapeutic opportunities against high-grade malignant gliomas? Oncologist. 2002;7(1):17-33.

18. Fischer U, Jänicke RU, Schulze-Osthoff K. Many cuts to ruin: a comprehensive update of caspase substrates. Cell Death Differ. 2003;10(1): 76-100.

19. Porter AG, Jänicke RU. Emerging roles of caspase-3 in apoptosis. Cell Death Differ. 1999;6(2):99-104.

20. Affar EB, Germain M, Winstall E, et al. Caspase-3-mediated processing of poly(ADP-ribose) glycohydrolase during apoptosis. J Biol Chem. 2001;276(4):2935-2942.

21. Drummond DC, Meyer O, Hong K, Kirpotin DB, Papahadjopoulos D. Optimizing liposomes for delivery of chemotherapeutic agents to solid tumors. Pharmacol Rev. 1999;51(4):691-743.

22. Allen TM, Cullis PR. Drug delivery systems: entering the mainstream. Science. 2004;303(5665):1818-1822.

23. Gabizon A, Catane R, Uziely B, et al. Prolonged circulation time and enhanced accumulation in malignant exudates of doxorubicin encapsulated in polyethylene-glycol coated liposomes. Cancer Res. 1994;54(4): 987-992.

24. Gabizon AA. Stealth liposomes and tumor targeting: one step further in the quest for the magic bullet. Clin Cancer Res. 2001;7(2):223-225.

25. Wang AZ, Langer R, Farokhzad OC. Nanoparticle delivery of cancer drugs. Annu Rev Med. 2012;63:185-198.

26. Ruoslahti E. Specialization of tumour vasculature. Nat Rev Cancer. 2002;2(2):83-90.

27. Brown JM, Giaccia AJ. The unique physiology of solid tumors: opportunities (and problems) for cancer therapy. Cancer Res. 1998;58(7): 1408-1416.

28. Tschaikowsky K. Protein kinase C inhibitors suppress LPS-induced TNF production in alveolar macrophages and in whole blood: the role of encapsulation into liposomes. Biochim Biophys Acta. 1994;1222(1): $113-121$. 
29. Yamauchi M, Kusano H, Nakakura M, Kato Y. Reducing the impact of binding of UCN-01 to human alpha1-acid glycoprotein by encapsulation in liposomes. Biol Pharm Bull. 2005;28(7):1259-1264.

30. Yost SE, Pastorino S, Rozenzhak S, et al. High-resolution mutational profiling suggests the genetic validity of glioblastoma patient-derived pre-clinical models. PLoS One. 2013;8(2):e56185.

31. Murphy EA, Majeti BK, Mukthavaram R, Acevedo LM, Barnes LA, Cheresh DA. Targeted nanogels: a versatile platform for drug delivery to tumors. Mol Cancer Ther. 2011;10(6):972-982.

32. Hewavitharana AK, Shaw PN, Ng YK, Fuerst JA. Simple screening method for staurosporine in bacterial cultures using liquid chromatography-tandem mass spectrometry. J Bioanal Biomed. 2009;1(1):001-004

33. Gurley LR, Umbarger KO, Kim JM, Bradbury EM, Lehnert BE. Development of a high-performance liquid chromatographic method for the analysis of staurosporine. J Chromatogr B Biomed Appl. 1995;670(1):125-138.

34. Charrois GJ, Allen TM. Drug release rate influences the pharmacokinetics, biodistribution, therapeutic activity, and toxicity of pegylated liposomal doxorubicin formulations in murine breast cancer. Biochim Biophys Acta. 2004;1663(1-2):167-177.

35. Kurata N, Kuramitsu T, Tanii H, et al. Development of a highly sensitive high-performance liquid chromatographic method for measuring an anticancer drug, UCN-01, in human plasma or urine. J Chromatogr B Biomed Sci Appl. 1998;708(1-2):223-227.

36. Wakimoto H, Kesari S, Farrell CJ, et al. Human glioblastoma-derived cancer stem cells: establishment of invasive glioma models and treatment with oncolytic herpes simplex virus vectors. Cancer Res. 2009;69(8):3472-3481.

37. Rajesh M, Sen J, Srujan M, Mukherjee K, Sreedhar B, Chaudhuri A Dramatic influence of the orientation of linker between hydrophilic and hydrophobic lipid moiety in liposomal gene delivery. J Am Chem Soc. 2007;129(37):11408-11420.

38. Page B, Page M, Noel C. A new fluorometric assay for cytotoxicity measurements in-vitro. Int J Oncol. 1993;3(3):473-476.

39. Jensen MM, Jørgensen JT, Binderup T, Kjaer A. Tumor volume in subcutaneous mouse xenografts measured by microCT is more accurate and reproducible than determined by $18 \mathrm{~F}-\mathrm{FDG}$-microPET or external caliper. BMC Med Imaging. 2008;8:16.

40. Gurley LR, Umbarger KO, Kim JM, Bradbury EM, Lehnert BE. High-performance liquid chromatographic analysis of staurosporine in vivo. Its translocation and pharmacokinetics in rats. J Chromatogr B Biomed Sci Appl. 1998;712(1-2):211-224.

41. Fuse E, Tanii H, Takai K, et al. Altered pharmacokinetics of a novel anticancer drug, UCN-01, caused by specific high affinity binding to alpha1acid glycoprotein in humans. Cancer Res. 1999;59(5):1054-1060.
42. Hoffman WP, Ness DK, van Lier RB. Analysis of rodent growth data in toxicology studies. Toxicol Sci. 2002;66(2):313-319.

43. Clarke SJ, Farrugia DC, Aherne GW, Pritchard DM, Benstead J, Jackman AL. Balb/c mice as a preclinical model for raltitrexed-induced gastrointestinal toxicity. Clin Cancer Res. 2000;6(1):285-296.

44. Iwamoto H, Torimura T, Nakamura T, et al. Metronomic S-1 chemotherapy and vandetanib: an efficacious and nontoxic treatment for hepatocellular carcinoma. Neoplasia. 2011;13(3):187-197.

45. Chang HI, Yeh MK. Clinical development of liposome-based drugs: formulation, characterization, and therapeutic efficacy. Int $J$ Nanomedicine. 2012;7:49-60.

46. Anderson M, Omri A. The effect of different lipid components on the in vitro stability and release kinetics of liposome formulations. Drug Deliv. 2004;11(1):33-39.

47. Chang F, Lee JT, Navolanic PM, et al. Involvement of PI3 K/Akt pathway in cell cycle progression, apoptosis, and neoplastic transformation: a target for cancer chemotherapy. Leukemia. 2003;17(3):590-603.

48. Mayo LD, Donner DB. A phosphatidylinositol 3-kinase/Akt pathway promotes translocation of Mdm2 from the cytoplasm to the nucleus. Proc Natl Acad Sci U S A. 2001;98(20):11598-11603.

49. Murphy EA, Majeti BK, Barnes LA, et al. Nanoparticle-mediated drug delivery to tumor vasculature suppresses metastasis. Proc Natl Acad Sci US A. 2008;105(27):9343-9348.

50. Tschaikowsky K, Brain JD. Staurosporine encapsulated into $\mathrm{pH}$ sensitive liposomes reduces tnf production and increases survival in rat endotoxin shock. Shock. 1994;1(6):401-407.

51. Munson JM, Fried L, Rowson SA, et al. Anti-invasive adjuvant therapy with imipramine blue enhances chemotherapeutic efficacy against glioma. Sci Transl Med. 2012;4(127):127ra36.

52. Drummond DC, Noble CO, Guo Z, Hong K, Park JW, Kirpotin DB. Development of a highly active nanoliposomal irinotecan using a novel intraliposomal stabilization strategy. Cancer Res. 2006;66(6): 3271-3277.

53. Stensrud G, Sande SA, Kristensen S, Smistad G. Formulation and characterisation of primaquine loaded liposomes prepared by a $\mathrm{pH}$ gradient using experimental design. Int J Pharm. 2000;198(2):213-228.

54. Niu G, Cogburn B, Hughes J. Preparation and characterization of doxorubicin liposomes. Methods Mol Biol. 2010;624:211-219.

55. Sadzuka Y, Takabe H, Sonobe T. Liposomalization of SN-38 as active metabolite of CPT-11. J Control Release. 2005;108(2-3):453-459.

56. Fernandes AS, Gonçalves AP, Castro A, et al. Modulation of fungal sensitivity to staurosporine by targeting proteins identified by transcriptional profiling. Fungal Genet Biol. 2011;48(12):1130-1138. 


\section{Supplementary material}

For physical characterization, the liposome suspension was diluted 1:10 in MilliQ water, phosphate-buffered saline, and $10 \mathrm{mM} \mathrm{NaCl}$. A $100 \mu \mathrm{L}$ aliquot was sized using light backscattering Zetasizer (ZEN 3600; Malvern Instruments, Malvern, UK, Supplementary Table 1). The same instrument measured particle net charge expressed in $\mathrm{mV}$. Size and surface zeta potential were obtained from three repeat measurements with a backscattering angle of $173^{\circ}$. Liposome morphology and size were further characterized using scanning electron microscopy (Supplementary Figure 1). Samples were prepared by applying $5 \mu \mathrm{L}$ droplets of the liposome suspension onto a polished silicon wafer. After drying the droplets at room temperature overnight, the wafer was coated with chromium, and then imaged on an XL-30 electron microscope (Philips, Eindhoven, the Netherlands) to $30,000 \times$. Although transmission electron microscopy would be expected to provide more morphologic detail, the scanning electron microscopy images shown here do confirm the spherical morphology of the liposomes, and dynamic light scattering measurements indicated an approximately $110 \mathrm{~nm}$ average diameter. For cell viability studies, all cells from
Table SI The size and zeta potential of the staurosporine liposomal particles in PBS (saline) and water measured by laser light scattering using a Malvern Zetasizer (Malvern Instruments, Malvern, UK) as described in the text

\begin{tabular}{lcl}
\hline & Size & Zeta \\
\hline Staurosporine liposomes (water) & $108 \pm 1.1$ & $2.18 \pm 2.4$ \\
Staurosporine liposomes (PBS) & $117.8 \pm 10.6$ & $-5.3 \pm 4.34$
\end{tabular}

Note: Values shown are the averages of the size and of ten zeta potential measurements (mean \pm standard deviation).

Abbreviation: PBS, phosphate-buffered saline.

A172, U118, U251, GBM4, GBM8, PC3, MDA-MB-231, and B16F10 lines were grown in 96-well plates in complete medium with $10 \%$ fetal bovine serum at $37^{\circ} \mathrm{C}$, and then either free drug or drug-loaded liposomes were added and the cells incubated for another 72 hours (Supplementary Figure 2). For liposomal staurosporine uptake and resultant cell morphology, U87 cells were labeled with green fluorescent protein and were plated onto 12-well plates. The cells were incubated with empty liposomes or staurosporine-loaded liposomes. All liposomes were formulated with BODIPY fluorescent labeling on the exterior lipid shell, and after incubation the cells were washed and imaged with a scanning confocal microscope (Nikon Corporation; Supplementary Figure 3).
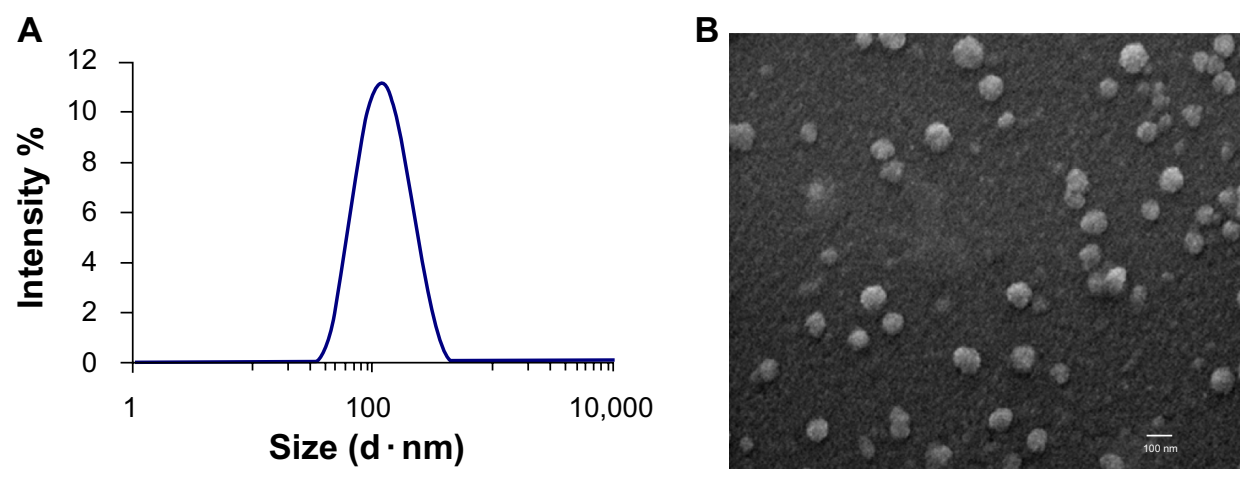

Figure SI Characterization of staurosporine liposomes by DLS and SEM. (A) Size (diameter, nm) of staurosporine liposomes measured by differential light scattering (DLS). (B) Representative scanning transmission electron microscope (SEM) images showing the structure of staurosporine liposomes. 
A

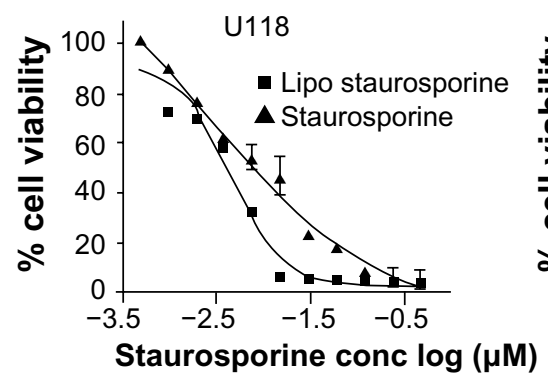

D

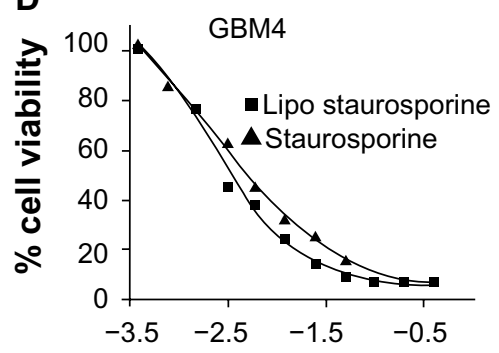

Staurosporine conc $\log (\mu \mathrm{M})$

G

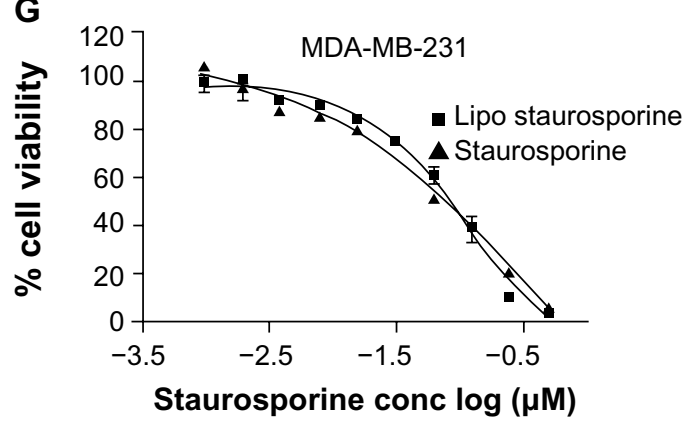

E

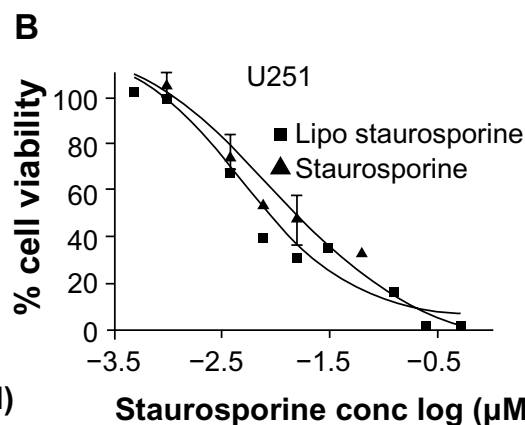

C
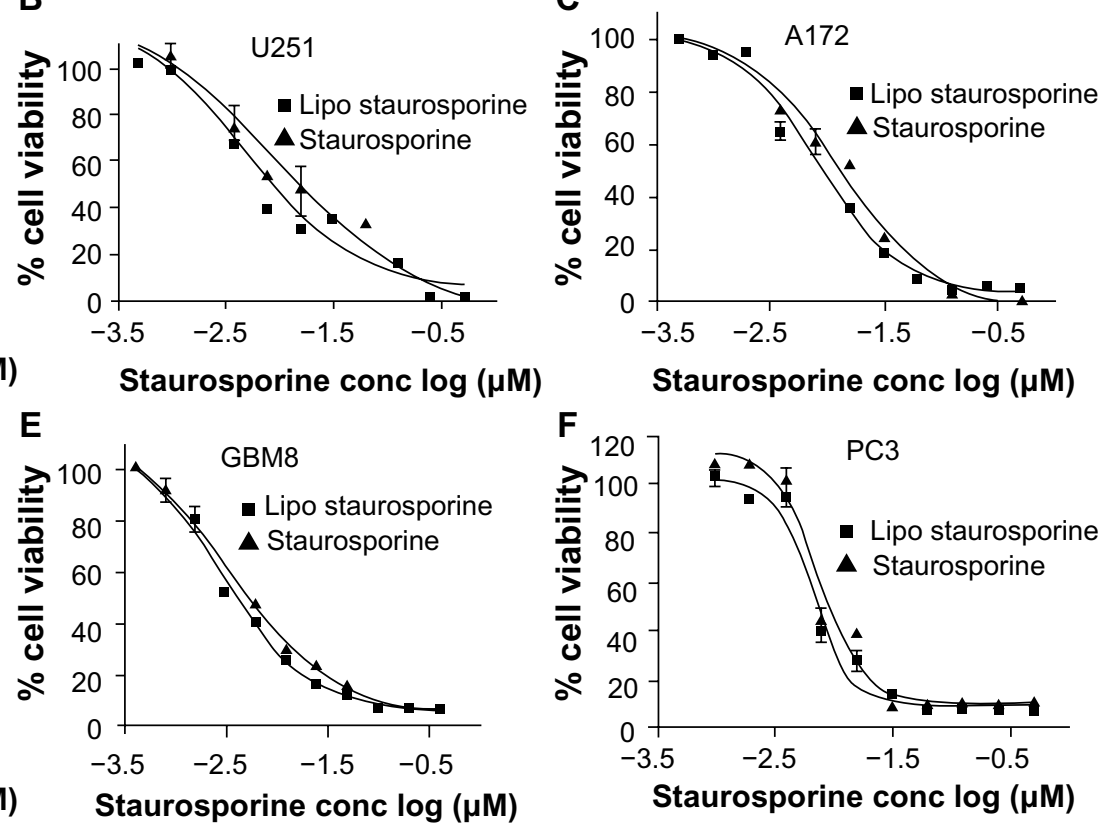

Figure S2 Graphs A-G depict staurosporine effects on viability (MTT assay) of the glioblastoma cell lines UII8 (A), U25I (B), AI72 (C), GBM4 (D), GBM8 (E) PC3 prostate tumor (F), and MDA-MB-23I breast tumor $(\mathbf{G})$. Experiments were performed in triplicate and the mean \pm standard error of the mean is indicated. Abbreviations: conc, concentration; Lipo, liposomal; MTT, (3-(4,5-dimethylthiazol-2-yl)-2,5-diphenyltetrazolium bromide. 


\section{Empty liposomes}
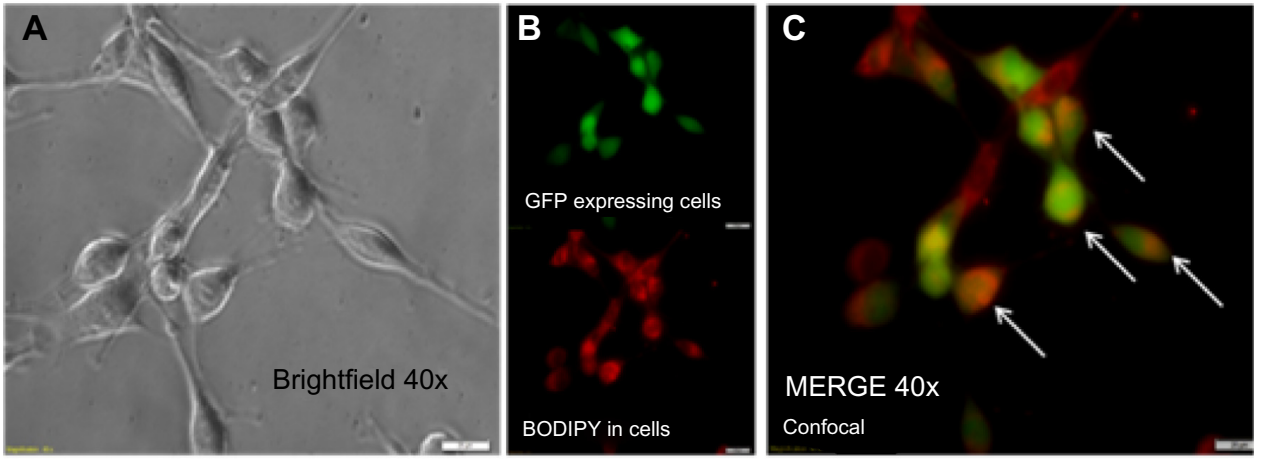

\section{Liposomes containing staurosporine}
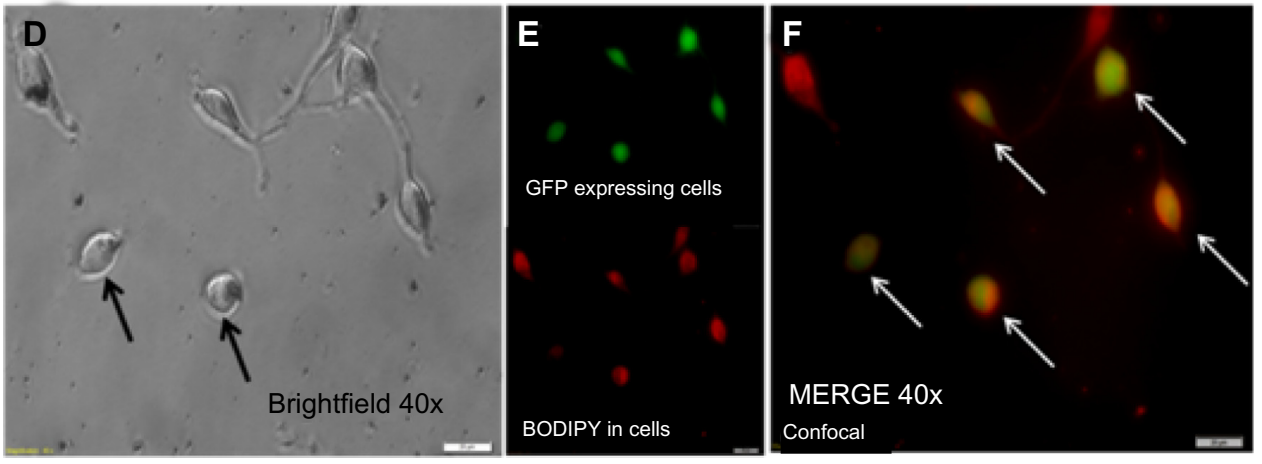

Figure S3 Liposomal uptake and morphological changes in U87 cells stably expressing GFP (green). Panel A is a brightfield image of the cells incubated with empty liposomes conjugated with fluorescent dye (BODIPY - red). Panel B shows these cells confocal imaged in the green channel (upper) and the red channel (lower). Panel C is a merge of green and red acquisitions and the arrows point to cells that clearly contain BODIPY labeled liposomes. Panel D is a brightfield image of U87 cells incubated with liposomes containing staurosporine. Note the rounded cells (arrows) which is consistent with apoptosis. Panel $\mathbf{E}$ shows the green and red channel acquisitions. Panel $\mathbf{F}$ is the merge. Note again the labeled liposomes are clearly inside the cells (arrows). In all images the white bars indicate $20 \mu \mathrm{M}$.

Abbreviations: GFP, green fluorescent protein; BODIPY, boron-dipyrromethene.

\section{Publish your work in this journal}

The International Journal of Nanomedicine is an international, peerreviewed journal focusing on the application of nanotechnology in diagnostics, therapeutics, and drug delivery systems throughout the biomedical field. This journal is indexed on PubMed Central, MedLine, CAS, SciSearch $\AA$, Current Contents ${ }^{\circledR} /$ Clinical Medicine,
Journal Citation Reports/Science Edition, EMBase, Scopus and the Elsevier Bibliographic databases. The manuscript management system is completely online and includes a very quick and fair peer-review system, which is all easy to use. Visit http://www.dovepress.com/ testimonials.php to read real quotes from published authors. 